Fetal Diagnosis and Therapy
Fetal Diagn Ther 2012;32:246-250

DOI: $\underline{10.1159 / 000338734}$
Received: December 6, 2011

Accepted after revision: April 5, 2012

Published online: August 22, 2012

\title{
Outcomes of Mid-Trimester Emergency Cerclage in Twin Pregnancies
}

\author{
Ishai Levin Liat Salzer Sharon Maslovitz Amiram Avni Joseph B. Lessing \\ Asnat Groutz Benny Almog \\ Department of Gynecology, Lis Maternity Hospital, Tel Aviv Sourasky Medical Center, Affiliated to the Sackler \\ School of Medicine, Tel Aviv University, Tel Aviv, Israel
}

\section{Key Words}

Emergency cerclage $\cdot$ Mid-trimester cerclage $\cdot$ Late cerclage

\begin{abstract}
Introduction: Placement of emergency cerclage at mid-trimester is controversial. At present, clinical trials comparing outcomes of mid-trimester cerclage to bed rest in twin pregnancies are lacking. Our aim was to examine the efficacy, safety and outcomes of mid-trimester cerclage in patients carrying twin gestations. Material and Methods: We retrospectively studied the outcomes of 14 patients carrying twin gestations with significant cervical dynamics who underwent cerclage. Outcomes of patients with cervical effacement only and patients with bulging membranes through the external os were examined. The interval between cerclage and delivery and the complication rates were calculated. Results: The average time interval between cerclage placement and delivery was 71.1 days overall. Patients with cervical shortening or effacement only $(n=10)$ gave birth an average of 80.2 days after the procedure, while 4 patients with bulging membranes gave birth an average of 48.5 days after cerclage placement. The overall procedure failure rate, defined as delivery before 28 completed weeks, was $14.2 \%$. Discussion: Patients carrying twin gestations with advanced
\end{abstract}

(C) 2012 S. Karger AG, Basel

$1015-3837 / 12 / 0324-0246 \$ 38.00 / 0$

Fax +4161306 1234

E-Mail karger@karger.ch

www.karger.com
Accessible online at: www.karger.com/fdt cervical changes might benefit from therapeutic cerclage. Further studies are required to demonstrate whether there is a difference compared to bed rest alone.

Copyright $\odot 2012$ S. Karger AG, Basel

\section{Introduction}

The role of second-trimester placement of cerclage is still controversial. Originally indicated for patients with an obstetrical history of mid-trimester painless dilatation, its use has now been extended to patients presenting with cervical effacement and dilatation (emergency cerclage). A significant amount of clinical research has dealt with the pros and cons of late emergency cerclage, given its high rates of complications. Some researchers were able to demonstrate benefits of cerclage placement even in cases of significant dilatation and membranes protruding through the external os $[1,2]$. The dilemma of cerclage placement is compounded by the heterogeneous characteristics of patients needing this intervention, such as singleton versus multiple gestations. As an example, Newman et al. [3] were not able to demonstrate a reduc-

I.L. and L.S. made equal contributions to the writing of this paper. 
tion in the risks of prematurity associated with cerclage placement in twin gestations with a shortened cervical length of $<25 \mathrm{~mm}$ measured by ultrasound. In another study, Roman et al. [4] were unable to demonstrate a lower incidence of spontaneous preterm delivery with cerclage as compared to conservative treatment in twin and triplet pregnancies. A study comparing the outcomes of late cerclage in twins with the twin-to-twin transfusion syndrome and treated with fetoscopic selective laser coagulation demonstrated improved outcomes after cerclage compared with bed rest [5].

As clinicians, we are sometimes faced with situations where there is limited evidenced-based data and the patients expect decisive action that is lacking. A patient with twin gestations presenting at mid-trimester with painless dilatation and bulging membranes is a clinical dilemma with a prognosis that seems dire, and as yet we still do not know if cerclage is the best solution. Our fear in such cases is primarily of causing a delivery at an extremely premature gestational week - after 23 weeks and 6 days and before 28 weeks. Survival before 24 weeks is anecdotal, and so most neonates do not survive. Neonates after 28 weeks usually have a good prognosis. Neonates between 23 weeks and 6 days and 28 weeks usually suffer severe long-term morbidity. It is these deliveries that we want to avoid.

In this case series, our primary aim was to report our results with emergency cerclage placement in patients with twin gestations presenting with significant effacement and dilatation at mid-trimester. This study is by no means a comparative study between cerclage and bed rest as our patients opted for intervention. As such we present the outcomes of late emergency cerclage in twin gestations for patients with cervical effacement and dilatation as well as results of the extreme condition of membranes protruding vaginally. Promising results would encourage us to conduct further studies comparing cerclage to bed rest, the current treatment of choice. Also, we would feel safer suggesting this type of operative intervention to patients presenting with such a clinical condition.

\section{Material and Methods}

Institutional ethics committee approval was given for this retrospective study. We retrospectively studied charts of patients with twin pregnancies admitted for cervical dynamics in mid-trimester. Data collection was carried out for patients admitted between October 1998 and September 2009. We collected demographic as well as gynecological and obstetrical data. Clinical examination of the cervix as well as cervical length measurements by ultrasound were recorded. Clinically evident cervical dynamics was defined as $50 \%$ effacement and over or estimation of cervical length below $2 \mathrm{~cm}$. On transvaginal ultrasound measurement, cervical shortening was defined as cervical length below $25 \mathrm{~mm}$. Due to the retrospective nature of this study, we could not record Bishop scores as these were not used or entered in the patients' charts.

Patients included in the study were patients with twin pregnancies of a minimum of 14 weeks +0 days. Patients were referred to the gynecological department by their treating physicians for clinically apparent cervical dynamics or because of symptoms such as abdominal pain or vaginal bleeding. All patients were examined, and cervical shortening was also verified by transvaginal ultrasound measurement. Before cerclage placement, all patients were hospitalized with bed rest for at least $24 \mathrm{~h}$. Infectious process was ruled out when no uterine activity was recorded, when there was no fever and when the patient was asymptomatic. Routine blood tests were taken to rule out leukocytosis as a marker of infection. For data recording purposes, we defined patients with clinical cervical dynamics as group 1 and patients with membranes bulging through the external os as group 2 .

All patients were started on oral amoxicillin and clavulanic acid (Augmentin) on admission and up to 7 days after cerclage. Cervical cerclage was placed under general anesthesia using the McDonald technique. After bladder drainage and cleansing with povidone-iodine $10 \%$ solution, the anterior and posterior lips of the cervix were grasped and a Mersilon cervical cerclage tape (Cervix-Set, Braun) was inserted at 4 points (upper and lower right and upper and lower left). The knot was fixed at the lower right point with long tails for easy removal. In cases of bulging membranes, we used the same technique but the membranes were gently pushed beyond the internal os with a Foley catheter inflated balloon. After cerclage, the balloon was deflated and gently removed. Tocolytic agents were not administered. Intraoperative techniques and complications were reviewed. We studied the complications of the procedures carried out as well as the time interval between the operative procedure and delivery and additional events following surgery. Pregnancy outcomes such as birth week, birth weights and Apgar scores were recorded. Because of the severe consequences of delivery of an extremely premature neonate, our definition of failure was delivery between 24 and 27 completed weeks (27 weeks +6 days), while success was defined as delivery beyond 28 completed weeks of gestation.

We analyzed the data for normal distribution using the Shapiro-Wilk test. As the data were normally distributed, we compared continuous variables using a t test and Fisher's exact test as appropriate. Logistic regression analysis was performed in order to identify predictors associated with cerclage success. Variables included in the regression analysis were the following: age, number of previous pregnancies, number of previous abortions, cervical length by ultrasound, bulging of membranes, clinical cervical length and gestational week. A p value of less than 0.05 was required to reject the null hypothesis.

\section{Results}

Between October 1998 and September 2009, we identified 14 patients with twin gestations who were treated with late emergency cerclage using the McDonald proce- 
Table 1. Demographic and obstetrical data

\begin{tabular}{llll}
\hline & All patients $(\mathrm{n}=14)$ & Group 1 $(\mathrm{n}=10)$ & Group 2 $(\mathrm{n}=4)$ \\
\hline Age, years & $31.1 \pm 6.5$ & $32.7 \pm 6.2$ & $27.6 \pm 6.1$ \\
Nulligravida & $12(85.7)$ & $9(90)$ & $3(75)$ \\
Previous early abortions & 5 patients had 1 & 3 patients had 1 & 2 patients had 1 \\
& 2 patients had 2 & & 2 patients had 2 \\
& 1 patient had 3 & $2(20)$ & $1(25)$ \\
Previous preterm had 3 & $3(21.4)$ & $2(2)$ & \\
\hline
\end{tabular}

Figures in parentheses represent percentages.

Table 2. Symptoms and outcomes

\begin{tabular}{llll}
\hline & All patients $(\mathrm{n}=14)$ & Group 1 $(\mathrm{n}=10)$ & Group 2 $(\mathrm{n}=4)$ \\
\hline Symptoms at presentation: pain/bleeding, $\mathrm{n}$ & $6 / 14$ & $4 / 10$ & $2 / 4$ \\
Gestation week at cerclage & $20.1 \pm 2.5$ & $19.6 \pm 2.8$ & $21.5 \pm 1.3$ \\
Cervical length, mm ${ }^{1}$ & $18.6 \pm 6.9$ & $19.4 \pm 4.4$ & $16.5 \pm 11.9$ \\
Time interval until delivery, days & $71.1 \pm 44.6$ & $80.2 \pm 45.3$ & $48.5 \pm 43.9$ \\
Delivery week & $30.7 \pm 6.5$ & $31.4 \pm 6.5$ & $29.0 \pm 7.2$ \\
Weight of 1st twin, g & $1,664.3 \pm 846.9$ & $1,692.4 \pm 798.7$ & $1,580.0 \pm 1,174.3$ \\
Weight of 2nd twin, g & $1,546.7 \pm 764.7$ & $1,650.3 \pm 777.1$ & $1,235.7 \pm 778.9$ \\
Chrioamnionitis, $\mathrm{n}$ & $3(21.4)$ & $2(20)$ & $1(25)$ \\
PPROM, n & $2(14.3)$ & $1(10)$ & $1(25)$ \\
Mortality, n & $7 / 28(25)$ & $4 / 20(20)$ & $3 / 8(37.5)$ \\
Preterm delivery $<24$ weeks, $\mathrm{n}$ & $2(14.3)$ & $1(10)$ & $1(25)$ \\
Preterm delivery $<28$ weeks, $\mathrm{n}$ & $2(14.3)$ & $1(10)$ & $1(25)$ \\
\end{tabular}

Figures in parentheses represent percentages. PPROM $=$ Preterm premature rupture of membranes. ${ }^{1}$ On ultrasound measurement.

dure. Ten patients had cervical shortening and dilatation or effacement as defined above (group 1), and 4 patients had cervical dilatation with membranes protruding through the external os to the vagina (group 2). Demographic as well as obstetrical data for the two groups of patients are summarized in table 1 . Among the group 1 patients, 2 were parents to one child each, but the women in the second group did not have children. All previous deliveries were spontaneous, without previous cesarean section. Among the patients in the first group, 2 patients had had a single previous late abortion at 22 and 23 weeks, respectively, and among the patients with bulging membranes (group 2), 1 patient had had a previous late abortion at 22 weeks. Twelve out of 14 patients in the two groups conceived after controlled ovarian stimulation or IVF for various indications.

Pregnancy and neonatal outcomes for the two groups combined and separately are summarized in table 2 .
Of the patients with cervical shortening (group 1), 1 patient had chorioamnionitis and delivered at 21 weeks of gestation, 14 days after cerclage placement, and 1 patient had preterm premature rupture of membranes with chorioamnionitis and delivered 64 days after cerclage placement at 19 weeks. One delivery in this group occurred at 27 weeks of a pair of twins weighing 759 and $872 \mathrm{~g}$.

Of the patients with bulging membranes (group 2), 1 patient had chorioamnionitis and delivered at 24 weeks (1 neonate survived), 19 days after cerclage placement, and 1 patient had chorioamnionitis and consequentially a late abortion at 22 weeks, 4 days after cerclage. Logistic regression analysis did not single out factors associated with cerclage success. In summary, 9 patients (64.3\%) delivered healthy babies after 28 weeks weighing 1,2442,830 and 1,044-2,700 $\mathrm{g}$ for the 1st and 2nd twin, respectively, all with Apgar scores of 9 or above. Average delivery week in this group was $34.8 \pm 3.8$. Three patients 
(21.4\%) had late abortions at 22-23 weeks, and 2 patients (14.3\%) delivered extremely premature neonates of 24 and 27 weeks. The overall mortality rate was $25 \%$ (7/28 neonates; table 2).

\section{Discussion}

In this study, we evaluated the efficacy and outcomes of mid-trimester cerclage placement in twin gestations. We evaluated outcomes for cerclage when cervical effacement is clinically apparent, and even in worse conditions, when dilatation and bulging of membranes occur. We found that patients can benefit from cerclage placement as the complication rate is low and the prognosis is good with only a $14.2 \%$ chance of delivery of an extremely premature neonate of below 28 weeks' gestation.

The benefits of mid-trimester cerclage placement in a singleton gestation have been the subject of wide research. In a large multicenter randomized study, Owen et al. [6] demonstrated benefits of mid-trimester cerclage placement for patients with singleton pregnancies and a shortened cervix of less than $25 \mathrm{~mm}$. In this study, women with a prior preterm birth and shortened cervix were less likely to have previable births. The perinatal mortality was also reduced, but preterm births of less than 35 weeks were not reduced, unless the cervical length was less than $15 \mathrm{~mm}$. In this large and well-designed study, the benefits of mid-trimester cerclage were apparent for singleton pregnancies with a shortened but not dilated cervix.

Placement of cerclage in worse scenarios of dilatation of the cervix and bulging membranes has also been examined [7]. Lipitz et al. [7] found a total mortality rate of $48.4 \%$ in a group of 32 patients carrying singleton pregnancies at mid-trimester with emergency cerclage. Perinatal mortality was significantly higher for patients with protruding membranes on admission. The mean interval from procedure to delivery was 7 weeks. In a similar study, Nelson et al. [8] demonstrated worse outcomes for mid-trimester cerclage placement in emergent cerclage (clinically indicated) as opposed to urgent (ultrasoundindicated) or elective (history-indicated) cerclage. In a retrospective cohort study of 99 patients who underwent mid-trimester cerclage, Debby et al. [2] demonstrated an overall neonatal survival of $82 \%$. Results were not significantly different when cerclage was placed with shortening or even with dilatation and bulging membranes. The time interval between procedure and delivery was statistically increased for patients without bulging membranes as opposed to patients with shortening of the cer-

Outcomes of Mid-Trimester Emergency

Cerclage in Twin Pregnancies vix only, being 14.3 versus 9.3 weeks, respectively. In fact, factors associated with successful mid-trimester placement of cerclage have also been studied [9]. Nulliparity, the presence of membranes protruding beyond the external os and gestational age of less than 22 weeks at the procedure were associated with a decreased chance of delivery at or beyond 28 gestational weeks.

The dilemma of mid-trimester placement of cerclage in twin gestations has also been examined. Roman et al. [4] found that sonographically indicated (closed cervix of length below $25 \mathrm{~mm}$ ) cerclage did not reduce the rate of spontaneous preterm birth as compared to no treatment in 414 sets of twins. Similar conclusions were found by Newman et al. [3], namely that mid-trimester cerclage in twins does not alter the risks of prematurity. However, both studies compared the results of cerclage placement to bed rest when the cervix was deemed short on ultrasound examination but not when patients presented with clinically apparent effacement. Moreover, Parilla et al. [10] found that patients with twin gestations and midtrimester cerclage placement who did not have a history of preterm delivery were more likely to deliver earlier than patients with no cerclage. These authors concluded that expectant management starting at 16-18 weeks seems more prudent. In their study, 17 patients with twins who needed emergent cerclage (cervical length of less than $15 \mathrm{~mm}$ with $>50 \%$ funneling) were identified, but no cases of cervical dilatation or membranes protruding through the external os were included [10].

This, in fact, was the aim of our study. The two studies cited above were conclusive in the respect that emergency cerclage is not beneficial when a short cervical length is demonstrated on ultrasound scan. Currently, most patients will be advised to remain in bed and rest throughout pregnancy in accordance with the papers cited above. Our aim was to examine whether mid-trimester emergency cerclage in extreme situations of cervical dynamics and twin gestations is in fact worthwhile. To date, this is the largest series of patients carrying twin gestations treated with emergency cerclage at mid-term due to clinically evident and significant cervical dynamics. Only a few reports in the literature exist to that effect. Benifla et al. [11] had 3 sets of twins in their study of emergency cerclage placement in extreme cervical conditions of dilatation or bulging membranes, but their outcomes for these twins were poor, with a delivery at 24 weeks in one set of twins.

The paucity of clinical data regarding emergency cerclage in twin gestations is also compounded by the fact that some twin pregnancies are dichorionic while others are monochorionic. Our study was underpowered to 
evaluate whether there is a difference in dichorionic compared to monochorionic twin pregnancies. Monochorionic twins are known to experience a substantially higher rate of complications, and it is possible that the use of cerclage could be of particular benefit in such cases [12].

As no guidelines exist regarding placement of cerclage for twins, and no comparative studies of cerclage versus bed rest exist, we aimed to determine whether this procedure was safe when performed in our patients and to estimate its success rate. As stated above, promising results would encourage us to conduct comparative studies of cerclage versus bed rest in such patients.

In essence, the main dilemma troubling the clinician is whether this procedure prolongs pregnancy into the viable neonatal range or whether complications will bring about delivery of an extremely premature neonate of between 24 and 27 completed weeks of gestation. Although pregnancy loss before 24 weeks is always a tragedy, we are rarely faced with neonates that survive. As such, the long-term neonatal morbidity is nonexistent. Also, neonates born after 28 weeks usually have good prognoses. The greatest tragedy in our belief is delivery at extreme neonatal prematurity, between 24 and 27 completed weeks, with severe long-term sequelae. Accordingly, it is of utmost importance to determine if cerclage placement in twin pregnancies under extreme cervical conditions and neonatal previability is beneficial to patients and neonates such that the time interval until delivery will be sufficient to prolong pregnancy to the viable and good prognosis range.
Our two groups of patients were similar with regard to demographic data, gynecological and obstetrical history and gestational age when the procedure was performed. Whereas patients with a shortened cervix had a mean interval of 80.2 days between procedure and delivery, patients with bulging membranes had a mean interval of 48.5 days until delivery.

In order to assess the true benefit of such a procedure, a prospective randomized study comparing treatment to bed rest should be undertaken, although obtaining the patients' approval for 'no intervention' would be difficult. Faced with an urgent situation of trying to rescue a pregnancy for which the prognosis seems poor, our patients will in all probability opt for treatment and not observation.

As stated above, the benefits in respect to observation are difficult to conclude, but we have demonstrated that clinically at least, emergency cerclage for this population is an option that may have a success rate of $64.4 \%$ for delivery beyond 28 weeks and a $14.2 \%$ chance of delivery of an extremely premature neonate. This information may help the clinician when consulting such patients.

We conclude that patients carrying twin gestations with advanced cervical changes might benefit from therapeutic cerclage. Further studies are required to demonstrate whether there is a difference compared to bed rest alone. Our encouraging results should act as the incentive to conduct a prospective comparative study of bed rest compared to mid-trimester emergency cerclage in twin gestations.

\section{References}

1 Althuisius SM, Dekker GA, Hummel P, van Geijn HP: Cervical incompetence prevention randomized cerclage trial: emergency cerclage with bed rest versus bed rest alone. Am J Obstet Gynecol 2003;189:907-910.

$\checkmark 2$ Debby A, Sadan O, Glezerman M, Golan A: Favorable outcome following emergency second trimester cerclage. Int J Gynaecol Obstet 2007;96:16-19.

- 3 Newman RB, Krombach RS, Myers MC, McGee DL: Effect of cerclage on obstetrical outcome in twin gestations with a shortened cervical length. Am J Obstet Gynecol 2002; 186:634-640.

4 Roman AS, Rebarber A, Pereira L, Sfakianaki AK, Mulholland J, Berghella V: The efficacy of sonographically indicated cerclage in multiple gestations. J Ultrasound Med 2005; 24:763-768; quiz 70-71.

\footnotetext{
Salomon LJ, Nasr B, Nizard J, Bernard JP, Essaoui $M$, Bussieres L, et al: Emergency cerclage in cases of twin-to-twin transfusion syndrome with a short cervix at the time of surgery and relationship to perinatal outcome. Prenat Diagn 2008;28:1256-1261.

6 Owen J, Hankins G, Iams JD, Berghella V, Sheffield JS, Perez-Delboy A, et al: Multicenter randomized trial of cerclage for preterm birth prevention in high-risk women with shortened midtrimester cervical length. Am J Obstet Gynecol 2009;201:375.e1-375.e8.

7 Lipitz S, Libshitz A, Oelsner G, Kokia E, Goldenberg M, Mashiach S, et al: Outcome of second-trimester, emergency cervical cerclage in patients with no history of cervical incompetence. Am J Perinatol 1996;13:419-422.

${ }_{8}$ Nelson L, Dola T, Tran T, Carter M, Luu H,

Terkildsen MF, Parilla BV, Kumar P, Grobman WA: Factors associated with success of emergent second-trimester cerclage. Obstet Gynecol 2003;101:565-569.

10 Parilla BV, Haney EI, MacGregor SN: The prevalence and timing of cervical cerclage placement in multiple gestations. Int J Gynaecol Obstet 2003;80:123-127.

-11 Benifla JL, Goffinet F, Darai E, Proust A, De Crepy A, Madelenat P: Emergency cervical cerclage after 20 weeks' gestation: a retrospective study of 6 years' practice in 34 cases. Fetal Diagn Ther 1997;12:274-278.

$>12$ Lewi L, Gucciardo L, Van Mieghem T, de Koninck P, Beck V, Medek H, et al: Monochorionic diamniotic twin pregnancies: natural history and risk stratification. Fetal Diagn Ther 2010;27:121-133.
} Dola C: Pregnancy outcomes following placement of elective, urgent and emergent cerclage. J Matern Fetal Neonatal Med 2009; 22:269-273. 